\section{Effect of pupil size on higher-order aberrations in high-myopic pseudophakic eyes with posterior staphyloma}

$X \mathrm{Zhu}^{1}, \mathrm{H} \mathrm{Ye}^{1}, \mathrm{~J} \mathrm{Yang}^{1}$ and $Y \mathrm{Lu}$
Department of

Ophthalmology, Eye and

Ear, Nose, and Throat

Hospital of Fudan University,

Shanghai, People's Republic of China

Correspondence:

Y Lu, Department of Ophthalmology, Eye and

Ear, Nose, and Throat

Hospital of Fudan

University, 83 Fenyang

Road, Shanghai 200031

People's Republic of China.

Tel: +86 02164377134 ;

Fax: +8602164318258.

E-mail: luyi_eent@126.com

${ }^{1}$ These authors contributed

equally to this work.

Received: 5 December 2013 Accepted in revised form:

10 September 2014

Published online: 17

October 2014

\begin{abstract}
Purpose To evaluate the effect of pupil size on higher-order aberrations (HOAs) in highmyopic pseudophakic eyes with posterior staphyloma using KR-1W aberrometer. Methods Forty eyes of 40 high-myopic cataract patients with posterior staphyloma and 20 eyes of 20 age-related cataract patients with normal axial length were recruited. At 1 month after phacoemulsification and intraocular lens implantation, ocular and internal HOAs as well as the visual quality data in the two groups were evaluated with KR-1W analyzer before and after mydriasis. Results Compared with normal axial length eyes, high-myopic pseudophakic eyes with posterior staphyloma had significantly lower values of internal coma both under 4-mm and 6-mm pupil sizes $(P<0.001$ and 0.020$)$, as well as internal total HOAs $(P<0.001)$ and third-order aberrations at 6-mm pupil size $(P=0.018)$. Statistically significant differences were also found in the variation from 4- to 6-mm pupil sizes for internal coma $(P=0.001)$, internal third-order aberrations $(P=0.009)$, internal total HOAs $(P=0.007)$, and ocular coma aberrations $(P=0.006)$ between two groups. After mydriasis, Strehl ratio increased significantly in high-myopic eyes, whereas decreased slightly in normal axial length eyes $(P=0.032$ when compared with the variation between the two groups). Statistically significant differences were also found for the variation of modulation transfer functions (MTFs) at frequencies of $15,30,45$, and 60 cycles/degree between the two groups $(P=0.002,0.002,0.004$, and 0.010 , respectively).
\end{abstract}

Conclusions High-myopic pseudophakic eyes with posterior staphyloma had significantly lower internal coma and less coma variations during mydriasis.

Eye (2015) 29, 98-105; doi:10.1038/eye.2014.242; published online 17 October 2014

\section{Introduction}

Nowadays, cataract surgery has changed from a visual rescue treatment to a refractive procedure. The patient's demand is not only limited to the improvement in visual acuity, but also better visual quality. Therefore, wavefront aberrations have become an important parameter for evaluating visual quality, ${ }^{1}$ among which higher-order aberrations (HOAs) are of great significance. It can represent part of the optical errors that lower order aberrations such as defocus and astigmatism cannot explain.

High myopia (HM) is a leading cause of visual impairment worldwide ${ }^{2}$ and its prevalence continues to increase, especially in Asian populations. ${ }^{3}$ Its definition lacks consensus, which is usually defined with the following features: long axial length $(\geq 26 \mathrm{~mm})$ or refractive error of $-6 \mathrm{D}$ or above $\mathrm{e}^{4}$ and pathological fundus changes such as posterior staphyloma, lacquer cracks, and chorioretinal atrophy. ${ }^{5}$ As the morphological characteristics of posterior staphyloma are now well understood after the application of optical coherence tomography, it is worthy to further look at the relationship between posterior staphyloma and visual quality of the highmyopic eyes. ${ }^{6}$ 
However, most of the previous studies regarding visual quality of high-myopic eyes were concerned with corneal or ocular HOAs, 7,8 and little was known about internal HOAs. In phakic eyes, internal HOAs, as part of the ocular HOAs, derive mainly from crystalline lens as well as other internal optic structure. ${ }^{9}$ However, in pseudophakic eyes, original structure of the eyes and intraocular lens (IOLs) are the major determinants of internal HOAs, which further affect the ocular HOAs. The intraocular structures of high-myopic eyes are largely different from those in eyes with normal AL. Therefore, choosing HM patients with posterior staphyloma as subjects for internal HOAs' research and making it clear about the relationship between certain intraocular structure and the HOAs would be of great importance.

Previous researches ${ }^{10-12}$ also showed that pupil size has an effect on HOAs. For postoperative cataract patients, this means that patients may obtain variable visual quality from day to night because of such HOAs' variations. Therefore, it is also meaningful to study the influence of pupil size on visual quality of high-myopic pseudophakic eyes with posterior staphyloma.

Up to now, there have been few studies on the internal HOAs due to lack of certain instruments. The KR-1W wavefront analyzer (Topcon, Tokyo, Japan), used in this study, combines wavefront and topography system, which can measure the ocular, corneal, and the internal HOAs simultaneously and rapidly. It achieves measurement of internal HOAs in a more accurate way, as the wavefront sensor and topographer are designed to function along same axis. This instrument employs the Hartmann-Shack method, based on which various aberrometers had achieved measurement of the aberrations in myopic eyes. ${ }^{13,14}$ Pupillometry is also integrated in this device, providing HOAs at both 4-mm and 6-mm pupil sizes, facilitating understanding the effect of pupil size on HOAs under different conditions.

With the KR-1W instrument, the current study aimed to investigate the effect of pupil size on HOAs in highmyopic pseudophakic eyes with posterior staphyloma and to identify whether this specific intraocular structure has potential impacts on internal HOAs.

\section{Materials and methods}

\section{Subjects}

Patients were selected from those who underwent eventful phacoemulsification and were implanted with IOLs (920H, Rayner, Hove, UK) with spherical aberration (SA) of $-0.18 \mu \mathrm{m}$ in our hospital between
November 2011 and May 2012. All the surgeries were carried out by one experienced surgeon. Sixty eyes of 60 cataract patients, aged from 45 to 80 years old, were involved in this study. Exclusion criteria were as follows: (1) ocular disorders, except for HM; (2) previous ocular surgeries other than phacoemulsification, such as corneal refractive surgery; (3) systemic diseases that might induce ocular disorders such as diabetes; and (4) intraoperative or postoperative complications, such as posterior capsule tear, persistent corneal edema, and posterior capsular opacification. This prospective study was performed according to the Declaration of Helsinki with provided written consent obtained from all the patients, after the nature of procedures has been explained clearly. All applicable institutional regulations concerning ethical use of human volunteers were followed during this research.

\section{Preoperative measurements}

Before surgery, routine ophthalmic examinations were carried out, including uncorrected visual acuity (UCVA), best-corrected visual acuity (BCVA), tonometry, B ultrasonography, and IOL power calculation using IOLMaster devices (Carl Zeiss, Oberkochen, Germany). According to the AL measured by IOLMaster, patients were divided into two groups: 20 eyes of 20 patients in normal AL group with AL $<24 \mathrm{~mm}$; and 40 eyes of 40 patients in HM group (all with posterior staphyloma) with $\mathrm{AL} \geq 26 \mathrm{~mm}$. The $\mathrm{B}$ ultrasonography results showed that all the HM patients had posterior staphyloma. Routine fundus examinations were carried out on all the patients and no obvious retinopathy such as severe chorioretinal atrophy, choroidal neovascularization, or macular hole was observed in both groups.

\section{Postoperative measurements}

At 1 month after surgery, postoperative examinations, including UCVA, BCVA, auto-refraction (Tonoref II, Nidek, Gamagori, Japan), anterior segment tomography (Pentacam HR, Oculus, Wetzlar, Germany), and wavefront aberrometry (KR-1W, Topcon), were carried out. The pupils were then dilated using a mixture of $0.5 \%$ phenylephrine and $0.5 \%$ tropicamide (Mydrin P; Santen Pharmaceutical Co., Osaka, Japan). After mydriasis, anterior segment tomography and wavefront aberrometry were performed again. Fundus examination was also carried out and no obvious retinal changes were found for both groups.

Pentacam system automatically carried out measurement and obtained 25 consecutive slit images of anterior segment. The system has a parameter-quality specification (QS) - to ensure the quality of the 
measurement, if patient is of poor fixation or blinks during the 2-s period of measurement, the QS is indicated with yellow or red color, and the data should not be used. During our measurement, all the subjects cooperated well without blink or poor fixation and were all qualified that QS was larger than 95\% (presented as 'OK'). Picking up the $0^{\circ}$ and $180^{\circ}$ frames from the 25 slit images of each eye, tilt and decentration of IOLs were also evaluated using ImagePro Plus software (Media Cybernetics, Inc, Rockville, MD, USA) as described previously, ${ }^{15}$ and no obvious tilt or decentration was observed (data not shown).

When performing wavefront aberrometry, patients were asked to stare at a focus and keep their eyes widely open for seconds. Measurement was performed automatically for three times and wavefront aberrations were displayed including total HOAs, trefoil, coma, spherical, third-order, and fourth-order aberrations of Zernike polynomials, calculated as root mean square values. The values of each measured order of ocular and internal aberrations were displayed simultaneously for both the central 4-mm and 6-mm pupil sizes. The aberrometer also provided the Strehl ratio obtained from point spread function (PSF) and MTF curves (for both $x$ and $y$ axes) as optical quality indexes of eyes, both of which were stimulated by $4-\mathrm{mm}$ size ocular wavefront Zernike coefficient. All measurements were carried out by the same trained optometrist in one dark room.

\section{Statistical analysis}

Definitions of the special terms used in this study were as follows:

$\Delta \mathrm{SE}$ (spherical equivalent $)=$ post-mydriasis $\mathrm{SE}-$ premydriasis $\mathrm{SE}$;

$\Delta \mathrm{HOAs}=$ post-mydriasis HOAs at 6-mm pupil

size - pre-mydriasis HOAs at 4-mm pupil size;

$\Delta$ coma $=$ post-mydriasis coma at 6-mm pupil

size - pre-mydriasis coma at 4-mm pupil size;

$\Delta$ Strehl ratio $=$ post-mydriasis Strehl ratio -

pre-mydriasis Strehl ratio; and

$\Delta \mathrm{MTF}=$ post-mydriasis $\mathrm{MTF}$ - pre-mydriasis MTF.

The values of all the parameters measured by KR-1W were means of three readings. Statistical analysis was performed using Stata/SE (10.0 version; StataCorp LP, College Station, TX, USA). Comparisons between preand post-mydriasis values of each HOA were performed by paired $t$-test, as well as comparisons of data between 4-mm and 6-mm pupil sizes. Comparisons of HOAs, $\Delta$ HOAs, $\Delta$ coma aberration, $\Delta S E$, and $\Delta$ Strehl ratio, as well as $\triangle \mathrm{MTF}$ values at each spatial frequency between normal AL group and HM group were performed by the independent two-sample $t$-test. It is considered as statistically significant when probability $(P)$ value $<0.05$.

\section{Results}

\section{Baseline characteristics}

Demographic characteristics of normal AL and HM groups are shown in Table 1 . No statistically significant differences were found for mean age, sex, ocular laterality, or $\triangle \mathrm{SE}$.

Before mydriasis, the average postoperative UCVA and BCVA for all the subjects was $0.432 \pm 0.204 \log$ MAR and $0.115 \pm 0.082 \log$ MAR, respectively. All the subjects had BCVA better than $0.300 \log$ MAR. As indicated in Table 1, statistically significant differences were identified in UCVA and SE between the normal AL and HM groups before mydriasis, whereas there was no statistically significant difference in BCVA between the two groups.

\section{Effects of mydriasis on ocular and internal HOAs}

Comparisons of ocular and internal HOAs between premydriasis 4-mm pupil size and post-mydriasis 6-mm pupil size were carried out on all the eyes. As shown in Figure 1, all the HOAs, except internal SA presented an increase in tendency (all $P<0.001$ ). No statistically significant differences were found for majority of the HOAs between pre- and post-mydriasis 4-mm sizes, except for ocular fourth-order and ocular SA (data not shown).

\section{Comparisons between normal AL and HM groups}

HOAs at specific pupil sizes Ocular and internal HOAs between normal AL and HM groups for pre-mydriasis

Table 1 Demographics between normal AL and HM groups

\begin{tabular}{lccc}
\hline & Normal AL group & HM group & P-value \\
\hline Eye $(n)$ & 20 & 40 & - \\
Age (years) & $63.4 \pm 4.8$ & $60.8 \pm 5.2$ & 0.540 \\
Sex (M/F) & $9 / 11$ & $17 / 23$ & 0.892 \\
Laterality (R/L) & $10 / 10$ & $22 / 18$ & 0.460 \\
$\Delta$ SE & $0.020 \pm 0.010 \mathrm{D}$ & $0.025 \pm 0.015 \mathrm{D}$ & 0.500 \\
UCVA & $0.358 \pm 0.182$ logMAR & $0.586 \pm 0.194 \log \mathrm{MAR}$ & 0.003 \\
BCVA & $0.108 \pm 0.078$ logMAR & $0.118 \pm 0.042 \log \mathrm{MAR}$ & 0.500 \\
SE & $-1.10 \pm 0.28 \mathrm{D}$ & $-2.50 \pm 0.65 \mathrm{D}$ & 0.012 \\
\hline
\end{tabular}

Abbreviations: AL, axial length; BCVA, best-corrected visual acuity; HM, high myopia; SE, spherical equivalent; UCVA, uncorrected visual acuity. $\Delta \mathrm{SE}=$ post-mydriasis $\mathrm{SE}$ - pre-mydriasis $\mathrm{SE}$; difference in age, $\triangle \mathrm{SE}$, and the postoperative UCVA, BCVA, and SE before mydriasis between normal $\mathrm{AL}$ and $\mathrm{HM}$ groups were compared using independent twosample $t$-test, and differences in sex and laterality were compared using $\chi^{2}$-test. 


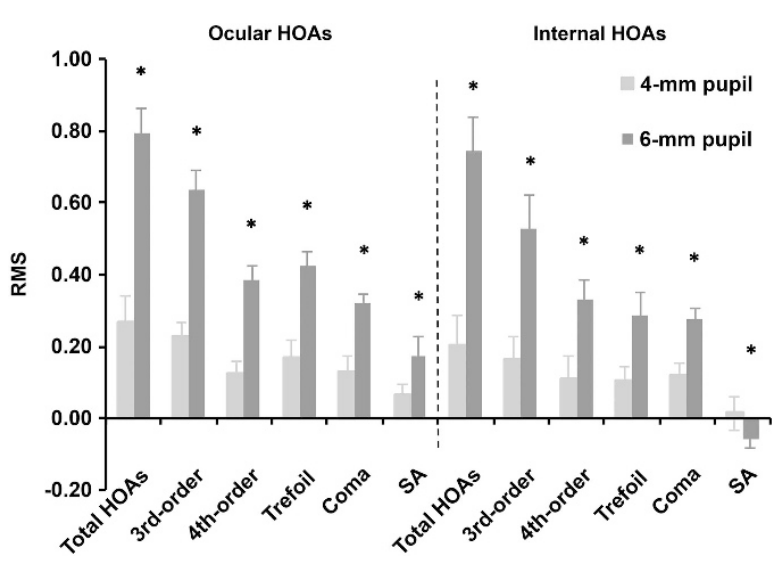

Figure 1 Ocular and internal aberrations of eye at premydriasis 4-mm pupil size and post-mydriasis 6-mm pupil size. Statistically significant differences were found for all the ocular and internal HOAs between pre-mydriasis 4-mm pupil size and post-mydriasis 6-mm pupil size. All the HOAs are calculated in root mean square values. ${ }^{*} P<0.001$ when compared between 4 -mm pre-mydriasis pupil size and $6-\mathrm{mm}$ post-mydriasis pupil size.

4-mm and post-mydriasis 6-mm pupil sizes were compared, respectively (Figure 2). For all the ocular HOAs and majority of the internal HOAs, there were no statistically significant differences between the two groups. However, compared with normal AL group, significantly lower values of internal coma aberrations were found for the HM group both at $4-\mathrm{mm}(P<0.001)$ and $6-\mathrm{mm}$ pupil sizes $(P=0.020)$. Differences in internal total HOAs and internal third-order aberrations at 6-mm pupil size between groups were also found statistically significant $(P<0.001$ and 0.018 , respectively).

$\triangle H O A s$ as a function of mydriasis To evaluate the function of mydriasis, comparisons for the $\Delta$ ocular and $\Delta$ internal HOAs between the two groups were also carried out. As indicated in Figure 3, statistically significant differences were found for $\Delta$ ocular coma $(P=0.006), \Delta$ internal total HOAs $(P=0.007), \Delta$ internal third-order aberrations $(P=0.009)$, and $\Delta$ internal coma ( $P=0.001$ ) between the two groups. The internal coma, third-order aberrations, and total HOAs increased more in normal AL group than HM group when pupil size enlarged from $4 \mathrm{~mm}$ to $6 \mathrm{~mm}$. However, the internal SA decreased after mydriasis (indicated as negative $\Delta$ value), while no statistically significant differences were found between groups.

Visual quality evaluation Before mydriasis, the Strehl ratios for normal AL and HM groups were $0.170 \pm 0.028$ and $0.158 \pm 0.043$; after mydriasis, the values were $0.150 \pm 0.024$ and $0.252 \pm 0.056$, respectively. As displayed in Figure 4, comparison of $\Delta$ Strehl ratio between normal
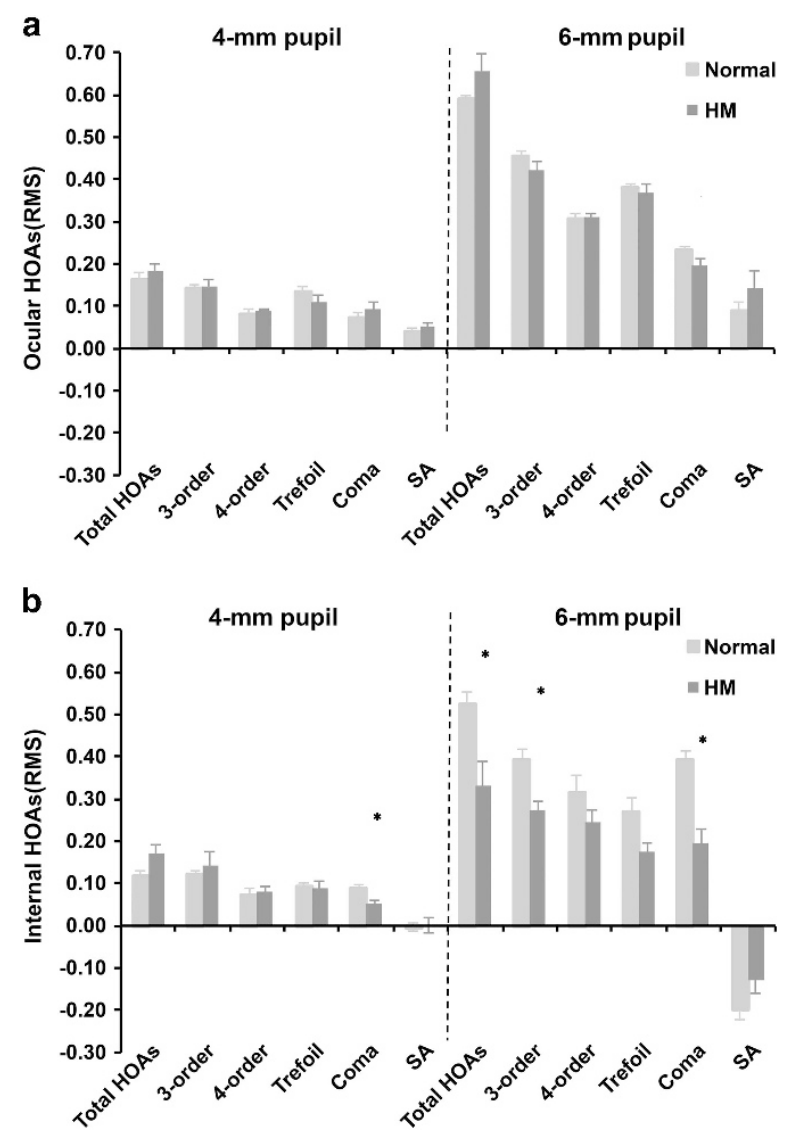

Figure 2 Comparisons of (a) ocular and (b) internal HOAs between normal axial length group and HM group both at premydriasis $4-\mathrm{mm}$ and post-mydriasis $6-\mathrm{mm}$ pupil sizes. There were no statistically significant differences between the two groups in the ocular HOAs and majority of the internal HOAs, whereas significantly lower values of internal coma aberrations were found for the HM group both at 4-mm and 6-mm pupil sizes, as well as internal total HOAs and internal third-order aberrations at 6 -mm pupil size. ${ }^{*} P<0.05$.

$\mathrm{AL}$ and HM groups was carried out, and the value for HM group was found significantly higher than that for normal AL group $(P=0.032)$. Strehl ratio improved markedly in HM patients as pupils were dilated. Figure 5 indicated that statistically significant differences were found at spatial frequencies of $15,30,45$, and 60 cycles/degree for $\triangle \mathrm{MTFs}$ between the two groups $(P=0.002,0.002,0.004$, and 0.010 , respectively, all $P>0.05$ at other spatial frequencies).

\section{Discussion}

The KR-1W is a new system that combines both wavefront sensor and topographer along the same axis. Compared with other diagnostic equipments that measure internal aberrations with special software, the special design of KR-1W could avoid the potential error 
a

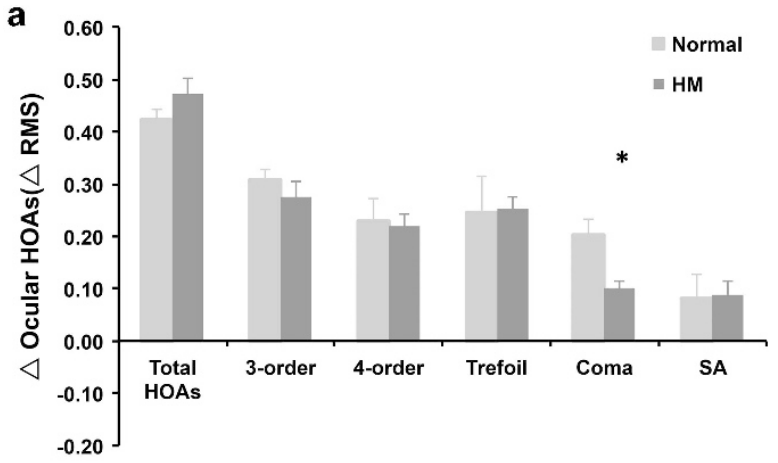

b

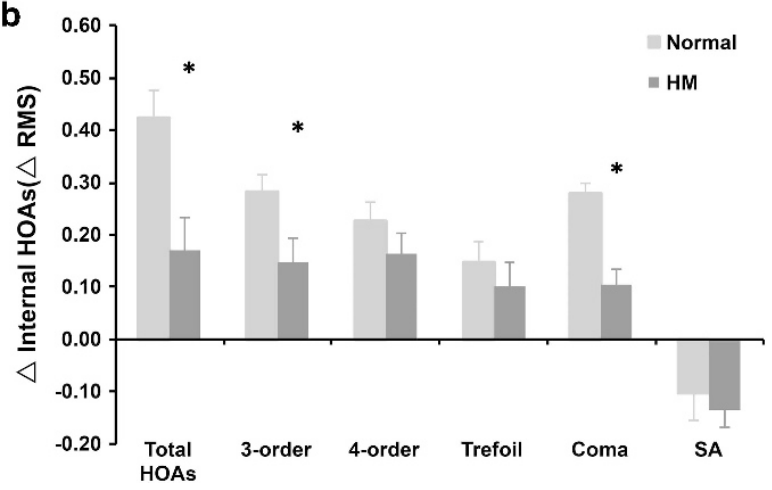

Figure 3 Comparisons of (a) $\Delta$ ocular HOAs and (b) $\Delta$ internal HOAs between normal axial length and HM groups. Statistically significant differences were found for $\Delta$ ocular coma, $\Delta$ internal total HOAs, $\Delta$ internal third-order aberrations, and $\Delta$ internal coma between the two groups. ${ }^{*} P<0.05$.

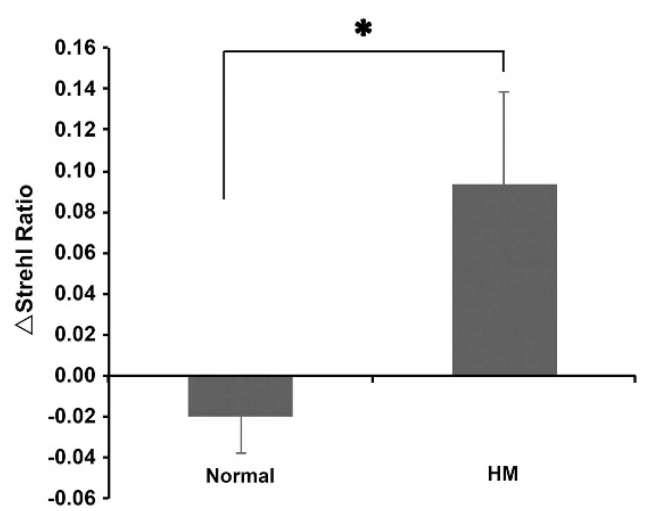

Figure 4 Comparison of $\Delta$ Strehl ratio between normal axial length (AL) and HM groups. After mydriasis, statistically significant increased Strehl ratio was found for HM group compared with that for normal AL group. ${ }^{*} P<0.05$. $\Delta$ Strehl ratio $=$ post-mydriasis Strehl ratio - pre-mydriasis Strehl ratio.

arising from axis misalignment. Research ${ }^{16}$ has proved good repeatability of KR-1W measurements for most of the HOAs. In the current study, with KR-1W analyzer, we measured the internal HOAs in pseudophakic highmyopic eyes with posterior staphyloma under different pupil sizes and found that posterior staphyloma might

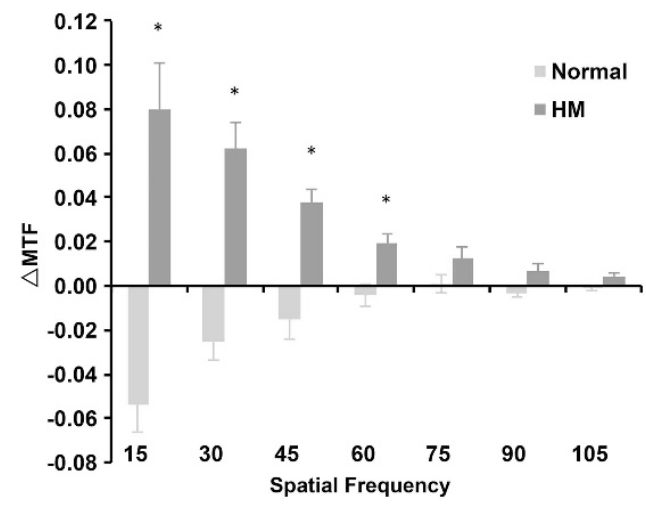

Figure 5 Comparison of $\triangle \mathrm{MTF}$ between normal axial length and HM groups. For spatial frequencies of 15, 30, 45, and 60 cycles/degree, statistically significant differences were found between groups. ${ }^{*} P<0.05$. $\triangle \mathrm{MTF}=$ post-mydriasis $\mathrm{MTF}-$ premydriasis MTF.

be the structure that offset the increased internal HOAs induced by mydriasis.

On the basis of previous researches, ,3,17,18 $\mathrm{HM}$ is of high prevalence in Asia, especially in China, and it affects people's visual function by large population both among children and adults. Relationship between HOAs and myopia is still under investigation. The result of Buehren ${ }^{7}$ study found increased aberrations in myopic groups, a positive correlation between spherical equivalent refractive error and aberrations were further identified; $;, 11$ some reported no relation between these two indices, ${ }^{19}$ other showed increased aberrations in hyperopic eyes compared with myopic eyes, especially for coma and SA. ${ }^{14}$ In pseudophakic eyes, we found that there were no statistically significant differences in the ocular total HOAs between the normal AL and HM groups either for the 4-mm or the 6-mm pupil sizes.

It is also noteworthy that HOAs involved in most of the studies were only about ocular and corneal ones, and internal HOAs were scarcely mentioned. HM eyes possess unique intraocular structures such as posterior staphyloma and so on, which will not change after cataract surgery, and would be potential contributors for their internal HOAs. Therefore, internal HOAs would be of great concern in HM eye study. We compared ocular and internal aberrations between pseudophakic normal $\mathrm{AL}$ group and HM group before and after mydriasis and found that internal coma aberration values of HM group were significantly lower than those of normal AL group both at 4-mm and 6-mm pupil sizes. Meanwhile, internal third-order aberrations and total HOAs at 6-mm pupil size were also lower in HM group, possibly due to its lower internal coma aberrations.

Internal aberration arises mainly from the intraocular structures. In this research, internal aberrations in the pseudophakic eyes were mainly induced by IOL and 
intraocular structures. Previous researches ${ }^{12,20}$ demonstrated that increased coma aberrations could be induced if tilt or decentration happens. The IOL position involved in our study was observed by Pentacam Scheimpflug System (Oculus) and no remarkable tilt or decentration was found in either normal AL or HM groups. Therefore, in HM eyes, there might be other intraocular factors rather than IOL that counteract the effect of coma aberrations.

Posterior staphyloma is a local protrusion of sclera in posterior pole of the eye, which is considered a distinguishing lesion in high-myopic eyes. The relative location of its ectasia area to the macula and optic nerve varies, according to which posterior staphylomas were classified. ${ }^{21}$ As coma aberrations derive from tilt or decentration of incident wavefront with respect to the optical surface, posterior staphyloma, throughout typical intraocular structures of high-myopic eye, would be of most probability correlated with coma aberration changes, affecting the wavefront that deviated on the misaligned optical surface. Therefore, we assumed that it would be the posterior staphyloma that caused the lower coma aberrations in high-myopic eyes compared with normal AL eyes.

As indicated in previous studies, ${ }^{10-12,22,23}$ increased pupil size was also found to increase the ocular aberrations. A similar phenomenon was observed in this study: all the ocular and internal HOAs were increased due to mydriasis, except internal SA, which might be neutralized by the implanted aspherical IOLs with negative SA, whose effects were much highlighted when pupil was dilated. To further identify if there are any differences in response to mydriasis between the normal AL and HM patients, we compared the variation of HOAs from 4-mm to 6-mm pupil size (defined as $\Delta$ HOAs in Materials and methods) between the two groups. The increasing amplifications were of no differences between the two groups for most of the HOAs, except for $\Delta$ internal coma, $\Delta$ internal third-order aberrations, and $\Delta$ internal total HOAs, as well as $\Delta$ ocular coma aberrations (see Figure 3). The increase in coma aberrations in HM group was less significant than that in normal AL group. As discussed above, we assumed that it was the posterior staphyloma that lowered the coma variation $(\Delta$ coma) induced by mydriasis in high-myopic eyes compared with normal eyes.

Variations in HOAs induce corresponding visual function changes. As an index of optical quality, Strehl ratio is closely associated with aberrations. ${ }^{24} \Delta$ Strehl ratio shows statistically significant difference between normal AL and HM groups in Figure 4. Negative $\Delta$ Strehl ratio in normal AL group suggested decreased optical quality after mydriasis, which was in accordance with previous studies, 7,24 whereas in HM group, positive $\Delta$ Strehl ratio was found, implying an improvement of optical function after mydriasis. As to the function of pupil size on MTFs, Lian $e t a l^{25}$ reported decreased MTF value for each measured spatial frequency with increasing pupil for emmetropic eyes, as well as for pseudophakic eyes after cataract surgery, ${ }^{26}$ which was similar to the trends observed in the normal AL group in the current study. However, in the HM group, all the $\triangle \mathrm{MTF}$ showed positive values, which might indicate a better visual function when pupil was dilated.

What needs to be mentioned was methods to evaluate PSF and MTF include double-pass technique, which is based on recording of the retinal image after double pass through the ocular media and retinal reflection, as well as to calculate and deduce from the wavefront results instead of direct measuring. The KR-1W applies the latter method, because all the eyes underwent no previous refractive surgery, values from both technologies would be similar.

As for normal AL eyes, the enlarged pupil size had a negative effect on optical function, whereas for highmyopic pseudophakic eyes, we discovered that a larger pupil brought about better visual function, it may lead to the following deduction that pupil size in high-myopic eyes might be larger than that in normal AL eyes to acquire better visual functions. This phenomenon was also observed in some previous studies, which discovered larger pupil diameter for myopia under photopia, $^{27}$ mesopic, ${ }^{28,29}$ or scotopic ${ }^{28}$ conditions. They considered this phenomenon as the effect of accommodation. Because less accommodation was required in myopic eyes than in hyperopic or emmetropic eyes, less pupillary constriction will be induced by the synkinesis between accommodation and pupil. In addition to their explanation, based on our findings in Figures 3, 4 and 5, we suspect that the better visual function caused by larger pupil in HM patients might also correlate with posterior staphyloma. However, further studies with larger sample sizes are still needed to make this hypothesis convincing, and aberrometers with different mechanisms might be applied. Meanwhile, evaluation of the posterior staphyloma morphology using optical coherence tomography might further demonstrate the role of this structure.

\section{Conclusions}

Using KR-1W, our study successfully measured internal aberrations in high-myopic pseudophakic eyes with posterior staphyloma. This special structure might have the effect of compensating the internal coma aberrations in HM patients, as well as the increased internal coma 
aberrations induced by mydriasis, which may consequently bring better visual qualities for highmyopic eyes under larger pupil size. Further studies are still needed to verify such effect.

\section{Summary}

What was known before

- When measuring HOAs, most previous researches focused on the ocular and corneal aberrations, and internal aberrations are still a novel field to discover.

- Possessing special intraocular structures, high-myopic eyes might have some corresponding HOAs' changes, whereas most of the previous studies were about high-myopic phakic eyes; HOAs for high-myopic pseudophakic eyes had seldom been studied.

What this study adds

- As a newly developed instrument, KR-1W achieves to measure internal aberration in a more accurate way.

- Using this instrument, our study achieved to measure internal aberrations for high-myopic pseudophakic eyes, which for the first time disclosed the potential effect of special internal structures on HOAs for HM patients.

- Based on the HOAs' data, it was identified that posterior staphyloma had compensative effect on internal coma aberrations, and this effect became more obvious when pupil enlarged, and brings about better visual quality.

- An interesting speculation would also for the first time be raised that high-myopic eyes would gradually develop into larger pupil size as a physiological compensation.

\section{Conflict of interest}

The authors declare no conflict of interest.

\section{Acknowledgements}

This work was supported by the National Natural Science Foundations of China (grant numbers: 81100653 and 81470613) and The Special Scientific Research Fund for Health Industry (number: 201302015).

\section{References}

1 Charman WN. Wavefront aberration of the eye: a review. Optom Vis Sci 1991; 68: 574-583.

2 Wong TY, Ferreira A, Hughes R, Carter G, Mitchell P. Epidemiology and disease burden of pathologic myopia and myopic choroidal neovascularization: an evidencebased systematic review. Am J Ophthalmol 2014; 157: 9-25.

3 Xu L, Li Y, Wang S, Wang Y, Wang Y, Jonas JB. Characteristics of highly myopic eyes: the Beijing Eye Study. Ophthalmology 2007; 114: 121-126.

4 He Miao, Wang Wei, Ragoonundun Dina, Huang Wenyong. Meta-analysis of the association between lumican gene polymorphisms and susceptibility to high myopia. PLoS One 2014; 9: e98748.

5 Morgan IG, Ohno-Matsui K, Saw SM. Myopia. Lancet 2012; 379: $1739-1748$.

6 Pruett RC. Complications associated with posterior staphyloma. Curr Opin Ophthalmol 1998; 9: 16-22.

7 Buehren T, Collins MJ, Carney LG. Near work induced wavefront aberrations in myopia. Vision Res 2005; 45: 1297-1312.

8 Wei RH, Lim L, Chan WK, Tan DT. Higher order ocular aberrations in eyes with myopia in a Chinese population. J Refract Surg 2006; 22: 695-702.

9 Wang L, Santaella RM, Booth M, Koch DD. Higher-order aberrations from the internal optics of the eye. J Cataract Refract Surg 2005; 31: 1512-1519.

10 Petermeier K, Frank C, Gekeler F, Spitzer MS, Messias A, Szurman P. Influence of the pupil size on visual quality and spherical aberration after implantation of the Tecnis 1-piece intraocular lens. Br J Ophthalmol 2011; 95: 42-45.

11 de Castro LE, Sandoval HP, Bartholomew LR, Vroman DT, Solomon KD. High-order aberrations and preoperative associated factors. Acta Ophthalmol Scand 2007; 85: 106-110.

12 McKelvie J, McArdle B, McGhee C. The influence of tilt, decentration, and pupil size on the higher-order aberration profile of aspheric intraocular lenses. Ophthalmology 2011; 118: 1724-1731.

13 Zhu XJ, Dai JH, Chu RY, Lu Y, Zhou XT, Wang L. Accuracy of WASCA aberrometer refraction compared to manifest refraction in Chinese adult myopes. J Refract Surg 2009; 25: 1026-1033.

14 Philip K, Martinez A, Ho A, Conrad F, Ale J, Mitchell P et al. Total ocular, anterior corneal and lenticular higher order aberrations in hyperopic, myopic and emmetropic eyes. Vision Res 2012; 52: 31-37.

15 Xing XJ, Tang X, Song H, Li WW. Comparison of tilt and decentration of four different kinds of aspheric intraocular lenses implantation. Zhonghua Yan Ke Za Zhi 2010; 46: 332-336.

16 Lopez-Miguel A, Martinez-Almeida L, Gonzalez-Garcia MJ, Coco-Martin MB, Sobrado-Calvo P, Maldonado MJ. Precision of higher-order aberration measurements with a new Placido-disk topographer and Hartmann-Shack wavefront sensor. J Cataract Refract Surg 2013; 39: 242-249.

17 Zhu XJ, Zhou P, Zhang KK, Yang J, Luo Y, Lu Y. Epigenetic regulation of $\alpha \mathrm{A}$-crystallin in high myopia-induced dark nuclear cataract. PLoS One 2013; 8: e81900.

18 Asakuma T, Yasuda M, Ninomiya T, Noda Y, Arakawa S, Hashimoto $S$ et al. Prevalence and risk factors for myopic retinopathy in a Japanese population: the Hisayama Study. Ophthalmology 2012; 119: 1760-1765.

19 Zadok D, Levy Y, Segal O, Barkana Y, Morad Y, Avni I. Ocular higher-order aberrations in myopia and skiascopic wavefront repeatability. J Cataract Refract Surg 2005; 31: 1128-1132.

20 Marcos S, Rosales P, Llorente L, Jimenez-Alfaro I. Change in corneal aberrations after cataract surgery with 2 types of aspherical intraocular lenses. J Cataract Refract Surg 2007; 33: 217-226.

21 Curtin BJ. The posterior staphyloma of pathologic myopia. Trans Am Ophthalmol Soc 1977; 75: 67-86.

22 Kasper T, Buhren J, Kohnen T. Intraindividual comparison of higher-order aberrations after implantation of aspherical and spherical intraocular lenses as a function of pupil diameter. J Cataract Refract Surg 2006; 32: 78-84. 
23 Kohnen T, Klaproth OK, Buhren J. Effect of intraocular lens asphericity on quality of vision after cataract removal: an intraindividual comparison. Ophthalmology 2009; 116: 1697-1706.

24 van den Bos A. Aberration and the Strehl ratio. J Opt Soc Am A Opt Image Sci Vis 2000; 17: 356-358.

25 Lian HF, Tang X, Wang J. The influences on modulation transfer function as a function of age and pupil in adult emmetropic eyes. Zhonghua Yan Ke Za Zhi 2010; 46: 227-232.

26 Santhiago MR, Netto MV, Barreto JJ, Gomes BA, Oliveira CD, Kara-Junior N. Optical quality in eyes implanted with aspheric and spherical intraocular lenses assessed by NIDEK OPD-Scan: a randomized, bilateral, clinical trial. J Refract Surg 2011; 27: 287-292.

27 Hashemi H, Yazdani K, Khabazkhoob M, Mehravaran S, Mohammad K, Fotouhi A. Distribution of photopic pupil diameter in the Tehran eye study. Curr Eye Res 2009; 34: 378-385.

28 Netto MV, Ambrosio RJ, Wilson SE. Pupil size in refractive surgery candidates. J Refract Surg 2004; 20: 337-342.

29 Hsieh YT, Hu FR. The correlation of pupil size measured by Colvard pupillometer and Orbscan II. J Refract Surg 2007; 23: 789-795. 